\title{
Influence of nutrition on prevention of diabetes mellitus
}

\author{
Bryndis Eva Birgisdóttir
}

\section{Introduction}

Environmental influences such as changes in lifestyle and nutritional factors are the most likely cause of the steep rise in incidence and prevalence of diabetes seen world-wide. The incidence of type 1 diabetes and the prevalence of type 2 diabetes are lower in Iceland than in the genetically related nations of the other Nordic countries, i.e. Norway, Denmark, Sweden and Finland. This indicates that there are environmental factors, for example, in the diet of the people in Iceland, that protect them against the disease. The aim of the thesis was to study the influence of nutrition on prevention of diabetes by investigating nutritionally related factors, i.e. cow's milk and size at birth, that may act protectively in the Icelandic population.

\section{Cow's milk and type I diabetes}

A debate regarding the possible role of cow's milk in the aetiology of type 1 diabetes has been ongoing for many years, as a relationship between cow's milk and type 1 diabetes is found in some areas but not others. The exposure to cow's milk in Iceland is not less than in the other Nordic countries, but the Icelandic bovine herd has been isolated for 1100 years, while breeding has been performed between and within herds in the other Nordic countries, changing the protein composition of the milk. Variation in the protein composition of cow's milk in different areas of the world may explain the inconsistency in studies investigating the relationship between type 1 diabetes and cow's milk.

The aim was to analyse Icelandic milk and milk from the other Nordic countries, as sold to consumers, for proteins suggested to be diabetogenic or protective against type 1 diabetes. The possible contribution of milk proteins to the lower incidence of type 1 diabetes in Iceland was investigated by studying the consumption of the proteins and the relationship between the consumption and incidence values.
Milk samples from the largest consumption areas in all Nordic countries were randomly collected and analysed for $\beta$-casein A1 and B as well as for bovine serum albumin, all suggested to be diabetogenic, and for immunoglobulin and lactoferrin, suggested to be protective. Consumption of A1 and $\mathrm{B} \beta$-casein in the countries was calculated among 2-year-old children and 11-14-year-old adolescents, using dietary studies from all of the Nordic countries. The per capita consumption of the other proteins was calculated using the FAOSTAT database. A retrospective case-control study for comparison of milk consumption in infancy was also performed in Iceland, including 55 subjects with type 1 diabetes and 165 controls.

A lower amount of A1 and B $\beta$-casein was found in Icelandic cow's milk than in cow's milk from the other Nordic countries $(p<0.02)$ (Fig. 1). The consumption of the proteins among 2-year-old children was also lower in Iceland than in the other Nordic countries, when calculated both as A1 $\beta$-casein $(p<0.05)$, and as the sum of A1 and B $\beta$-casein $(p<0.05)$. A correlation was found between the amount of A1 $\beta$-casein consumed in this age group and the incidence of type 1 diabetes in the Nordic countries $(r=0.9 ; p<0.05)$. However, a correlation was not found for the sum of A1 and B $\beta$-casein. No statistical difference in the amount consumed or correlation with incidence values was found for consumption of the proteins among the adolescents $(p>0.05)$. The highest amount of bovine serum albumin was found in Icelandic milk and the per capita consumption of this protein was higher in Iceland than in the other Nordic countries $(p<0.05)$, which was not expected for a protein proposed to be diabetogenic. The consumption of immunoglobulin was also higher in Iceland $(p<0.05)$, while there was no difference in the consumption of lactoferrin $(p>0.05)$. However, there was no correlation between the amount consumed of each of these three proteins and the incidence of type 1 diabetes in the countries studied 
$(p>0.05)$. Introduction of cow's milk products was similar among type 1 diabetes patients and controls in Iceland $(p>0.1)$.

The results indicate that lower amounts of A1 and B $\beta$-casein found in Icelandic cow's milk, resulting in lower consumption of the proteins among young children, may explain the lower incidence of type 1 diabetes in Iceland than in the other Nordic countries. Per capita consumption of bovine serum albumin, immunoglobulin or lactoferrin did not seem to explain the lower incidence of type 1 diabetes in Iceland. Cow's milk consumption in infancy was not related to type 1 diabetes in Iceland. The results show that cow's milk may vary in diabetogenicity depending on its protein composition. In the future, making cow's milk free of diabetogenic factors may help in the battle against the increasing incidence of type 1 diabetes. However, more studies are urgently needed on the effects of varying cow's milk proteins on human health.

\section{Weight gain in pregnancy, size at birth and type 2 diabetes}

The environmental factor most often associated with type 2 diabetes is adult overweight and obesity, but the Icelandic adult population does not weigh less than the nations of the other Nordic countries. Babies in Iceland, however, weigh more at birth than babies in the other Nordic countries, which may be of importance regarding the lower prevalence of type 2 diabetes in Iceland, as studies in other countries have found a positive relationship between low birth weight and later diseases such as type 2 diabetes. Women's weight gain in pregnancy is associated with their babies' size at birth. Recommendations on weight gain have varied over the last few decades and have been lower than the mean weight gain in pregnancy among Icelandic women. Regarding high weight gain in pregnancy, there has been concern over later overweight and obesity of the mother and complications in pregnancy and delivery.

The aim was to investigate the relationship between babies' size at birth and glucose intolerance in adulthood in a high birth weight population and the relationship between babies' size at birth and mothers' weight gain in pregnancy among women of normal weight before pregnancy. The relationship between weight gain in pregnancy and complications in pregnancy and delivery as well as later maternal overweight was also studied to be able to make recommendations regarding optimal weight gain in pregnancy for this group.

Information on birth size was collected from midwives' birth records at the National Archives, and information on adult size and glucose intolerance came from the Icelandic Heart Association

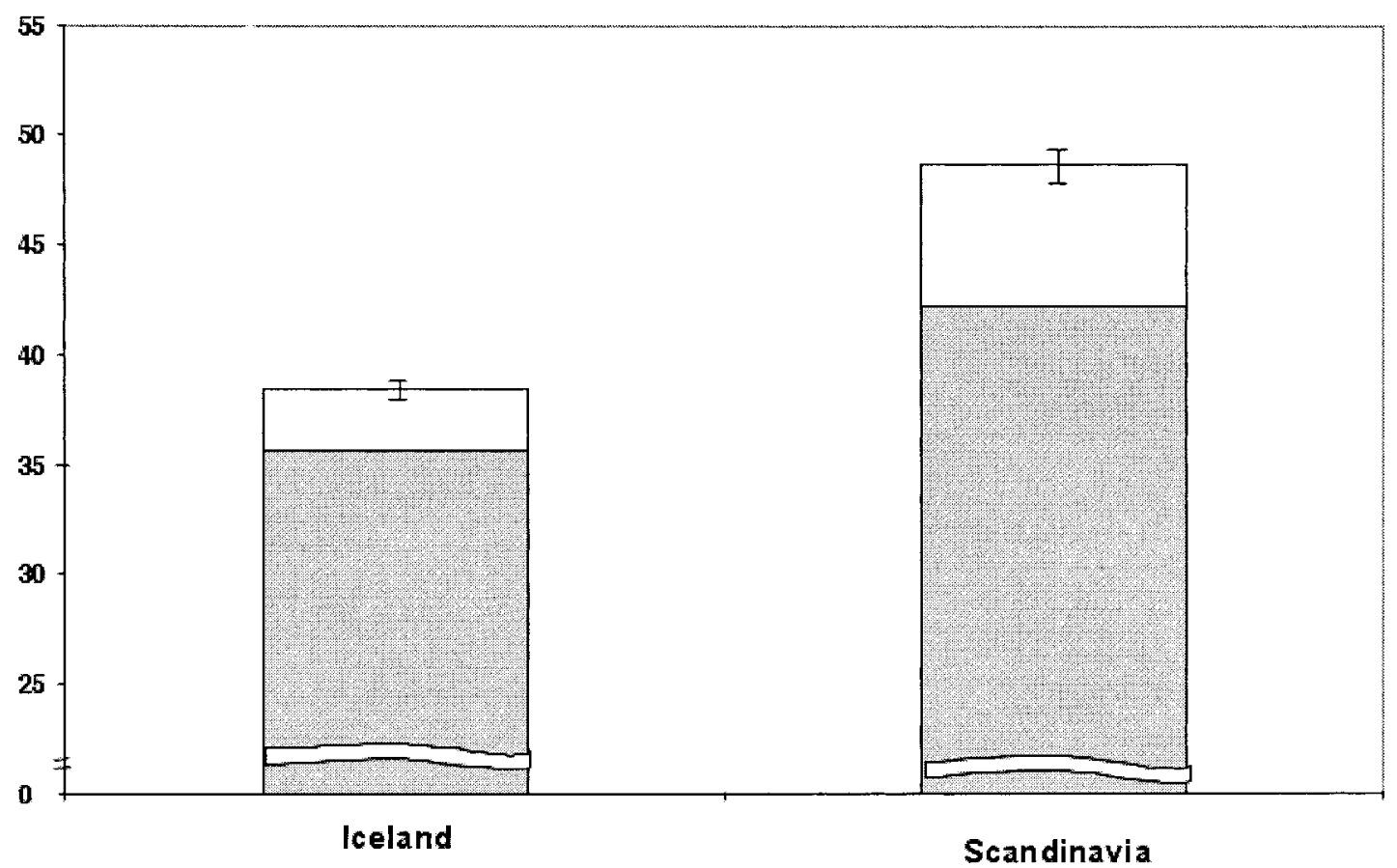

Fig. 1. Fractions (mean and SE) of the sum of A1 (dark shading) and B (light shading) $\beta$-casein found in milk from Iceland and the other Nordic countries (here as Scandinavia) $(p<0.02)$. 

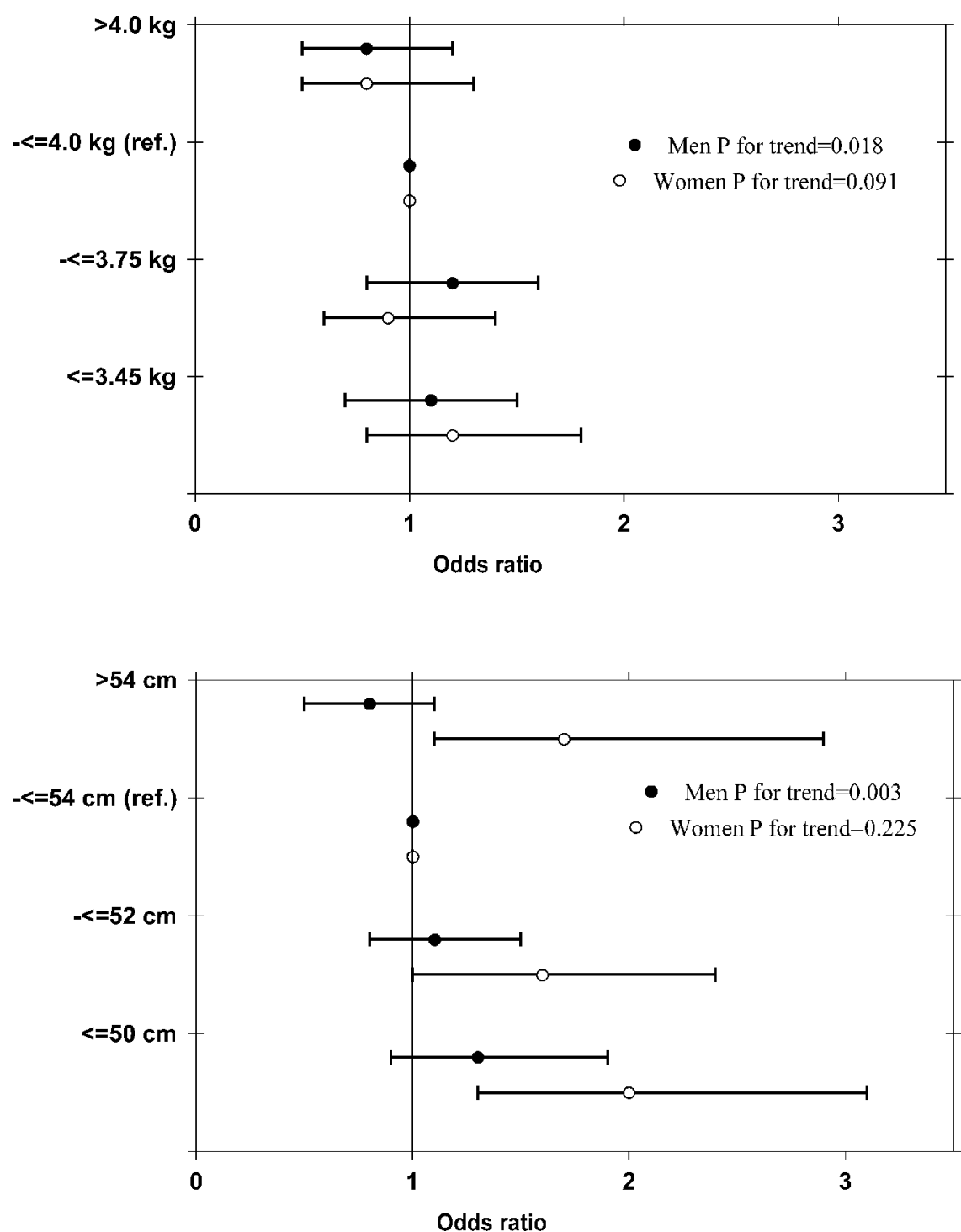

Fig. 2. Odds ratio and $95 \%$ confidence intervals, for dysglycaemia, i.e. impaired glucose tolerance or type 2 diabetes, by quartiles of birth weight (top) and length (bottom) adjusted for body mass index.

randomized prospective Reykjavik Study. Subjects were 4648 men and women aged 33-65 years. For the studies on weight gain in pregnancy, maternal information and information on babies' birth size were collected from maternity records (total $n=$ 800), at the Landspitali-Universit y Hospital and by telephone interviews.

In the study on the relationship between size at birth and glucose intolerance, postchallenge glucose was inversely related to birth weight and length for men $(p<0.001)$ and inversely related to birth weight and ponderal index for women $(p<$ $0.001)$. This association was mainly found among those within the highest third of adult body mass index. When classifying the data as dysglycaemia (impaired glucose tolerance or type 2 diabetes) or not, a lower prevalence was seen for men with increasing weight $(p<0.05)$ and length $(p<0.005)$ at birth but not with ponderal index. For women, there was no linear trend for dysglycaemia with size at birth, but the relation with birth length was U-shaped (Fig. 2). In the studies on weight gain in pregnancy, a low weight gain $(<11.5 \mathrm{~kg})$ was associated with an increased frequency of infants weighing less than $3.5 \mathrm{~kg}$ at birth $(p<0.01)$. When divided in quintiles, a relative risk of $2.69(95 \%$ confidence interval $(1.01-7.18, p<0.05)$ was found for complications in pregnancy among those gaining $>17.8-20.8 \mathrm{~kg}$ in pregnancy, compared with those gaining $12.5-15.5 \mathrm{~kg}$ (reference group). No statistically significant increase in risk was seen for those gaining $>15.5-17.8 \mathrm{~kg}(p>0.05)$ compared 
with the reference group. Most women $(88.6 \%)$ had regained their normal weight $18-24$ months postpartum despite gaining up to $24 \mathrm{~kg}$ in pregnancy. Women of normal weight before pregnancy reached normal weight again irrespective of gestational weight gain.

The results indicated a protective effect of increased size at birth with regard to glucose intolerance in adulthood in a population of greater birth size than previously investigated. The association was enhanced among those overweight or obese as adults. The results also indicated that low weight gain in pregnancy should be avoided to optimize birth outcome. A weight gain in pregnancy of $11.5-18 \mathrm{~kg}$ among women of normal weight before pregnancy gave the lowest risk for pregnancy and delivery complications and was not related to overweight or obesity postpartum. Sound, scientifically based recommendations regarding weight gain in pregnancy, which aim to secure the health of the mother and child, will help to decrease the number of low birth weight babies. This may help in the battle against the increasing prevalence of type 2 diabetes world-wide.

\section{Articles in Thesis}

1. Thorsdottir I, Birgisdóttir BE: Different weight gain in women of normal weight before pregnancy, postpartum weight and birth weight. Obstet Gynecol 1998;92:37783.

2. Thorsdottir I, Birgisdóttir BE, Johannsdottir IM et al: Different beta-casein fractions in Icelandic vs. Scandina- vian cow's milk may influence diabetogenicity of cow's milk in infancy, and explain low incidence of insulin-dependent diabetes mellitus (IDDM) in Iceland. Pediatrics 2000;106:719-24.

3. Thorsdottir I, Torfadottir JE, Birgisdóttir BE, Geirsson RT: Weight gain in women of normal weight before pregnancy: complications in pregnancy or delivery and birth outcome. Obstet Gynecol 2002;99:799-806.

4. Birgisdóttir BE, Gunnarsdottir I, Thorsdottir I et al: Size at birth and glucose intolerance in a relatively genetically homogenous, high birth weight population. Am J Clin Nutr 2002;76:399-403.

5. Birgisdóttir BE, Hill JP, Harris DP, Thorsdottir I. Variation in consumption of cow milk proteins and lower incidence of type 1 diabetes in Iceland versus the other 4 Nordic countries. Diabetes Nutr Metab 2002;15:240245.

6. Birgisdóttir BE, Hill J, Thorsson AV, Thorsdottir I. Children's rather than adolescents' lower consumption of the cow milk protein A1 beta-casein may explain the lower incidence of type 1 diabetes in Iceland than Scandinavia. (Submitted).

The article is based on the author's PhD thesis, which was conducted at the Unit for Nutrition Research, Landspitali-University Hospital and University of Iceland. Defended on June 15, 2002. ISBN: 9979-9516-2-।

Bryndis Eva Birgisdóttir

Unit for Nutrition Research

Lanspitali-University Hospital and University of Iceland

Eiriksgata 29

IS-I0 I Reykjavik

Iceland

E-mail: bryndise@landspitali.is 